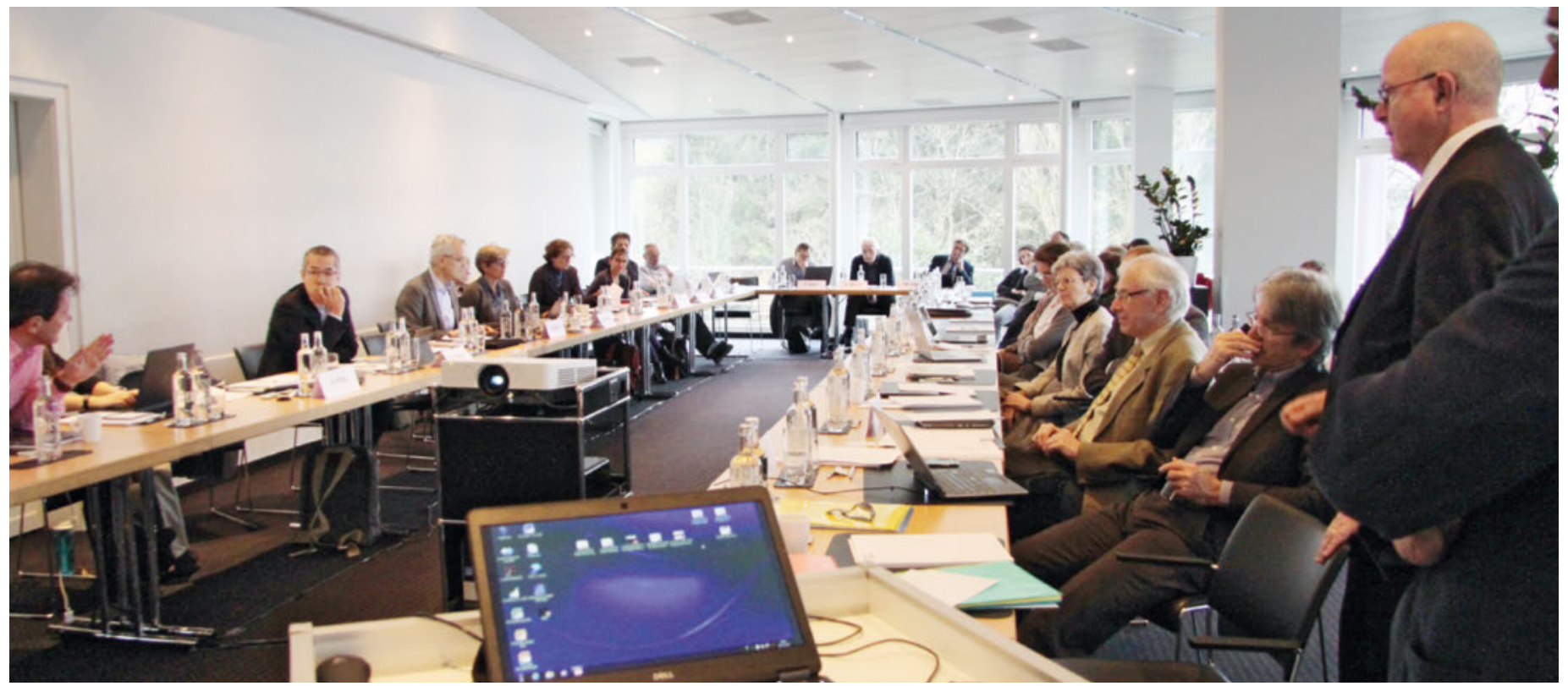

\title{
Durchblick in der Bildung
}

\section{Adrian Ritter}

Freier Journalist

Wie weiter mit der ärztlichen Bildung? Dieser Frage widmet sich die jährliche Journée de réflexion. Zu reden gaben Ende Januar vor allem der Ärztemangel und die zu grosse Distanz zwischen ärztlicher Ausbildung und Weiterbildung.

«Mit Seeblick zum Durchblick»: Unter diesem Motto veranstalten das Schweizerische Institut für ärztliche Weiter- und Fortbildung (SIWF) und das Collège des

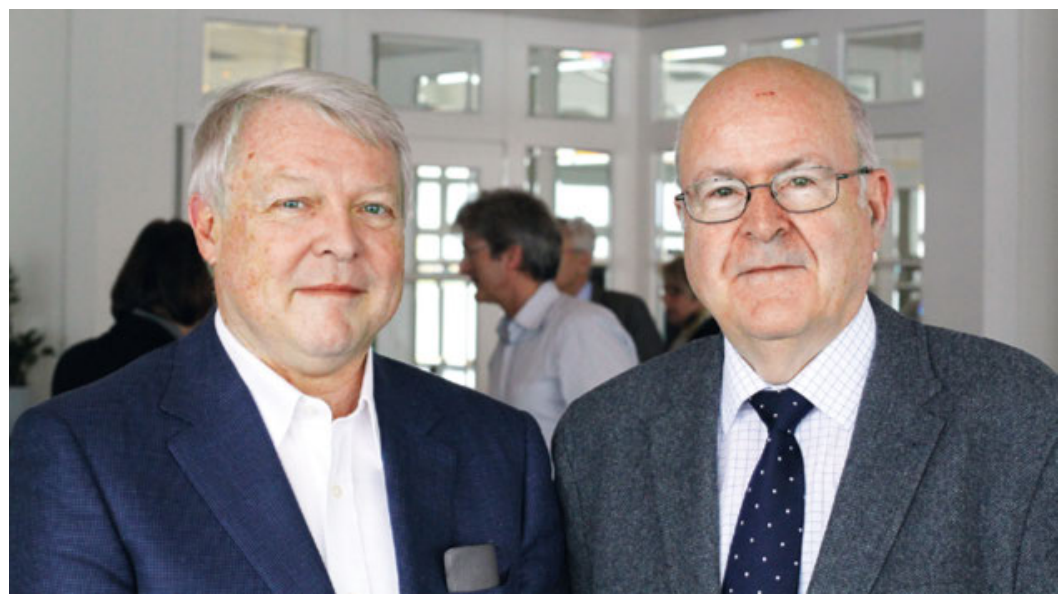

Moderierten die Journée de réflexion: Henri Bounameaux (links), Präsident des Collège des Doyens, und Werner Bauer, Präsident des Schweizerischen Instituts für ärztliche Weiter- und Fortbildung (SIWF).
Doyens (Kollegium der Dekane) jährlich die Journée de réflexion in der Nähe von Luzern. Beim Seeblick ist demnach der Vierwaldstättersee gemeint. Beim Durchblick geht es darum, die Gegenwart und die Zukunft der ärztlichen Bildung zu reflektieren. Rund 30 Personen nahmen an der diesjährigen Veranstaltung teil. Moderiert wurde sie von SIWF-Präsident Werner Bauer und Henri Bounameaux, Präsident des Collège des doyens. Werner Bauer stellte bei der Begrüssung klar: «Das Medizinstudium sowie die Weiter- und Fortbildungsangebote in der Schweiz sind exzellent. Deren Qualität ist aber weder selbstverständlich noch garantiert.» Die Herausforderungen seien zahlreich, um nur einige zu nennen: Der ökonomische Druck erhöhe das Spannungsfeld der Spitäler zwischen Lehranstalt und Dienstleistungsbetrieb, die Digitalisierung schaffe neue Berufsbilder und die Lernziele seien immer wieder auf ihren Realitätsbezug zu prüfen. Zudem müsse die Finanzierung der Weiterbildung neu diskutiert werden. 


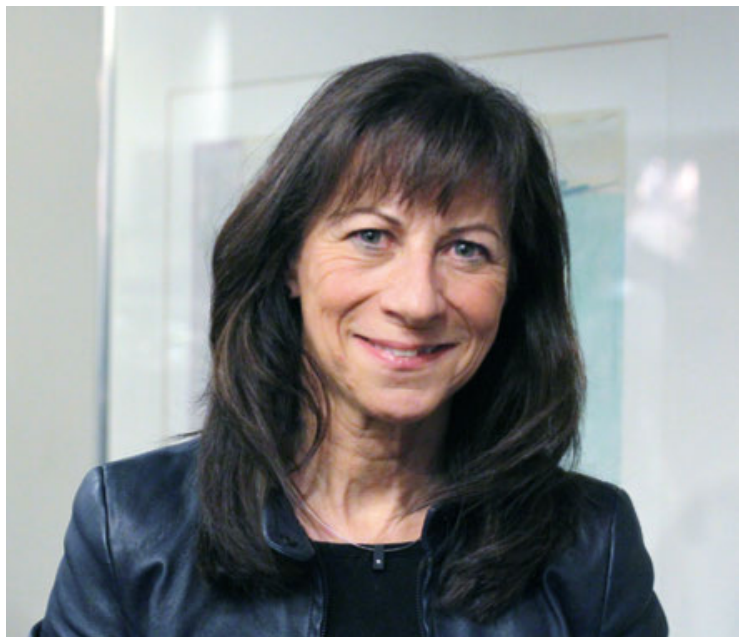

Referierte über die Finanzierung der ärztlichen Aus- und Weiterbildung: Regierungsrätin Heidi Hanselmann.

\section{Solidarität mit Grenzen}

Was die Finanzierung anbelangt, berichtete Regierungsrätin Heidi Hanselmann in ihrem Referat von interessanten Entwicklungen. Hanselmann ist Gesundheitsdirektorin des Kantons St.Gallen und Vizepräsidentin der Gesundheitsdirektorenkonferenz (GDK). «Das Ziel muss es sein, die Finanzierung der ärztlichen Ausbildung und Weiterbildung solidarischer zu gestalten - indem sich auch Kantone ohne medizinische Fakultät daran beteiligen", sagte Hanselmann.

Mit dieser Idee hatten sieben Kantone der Ostschweiz 2011 aus eigenem Antrieb beschlossen, sich an den Kosten für universitäre Lehre und Forschung zu beteiligen. Die Absicht war, mittelfristig eine gesamtschweizerische Lösung zu finden, unter Berücksichtigung der Finanzkraft der einzelnen Kantone.

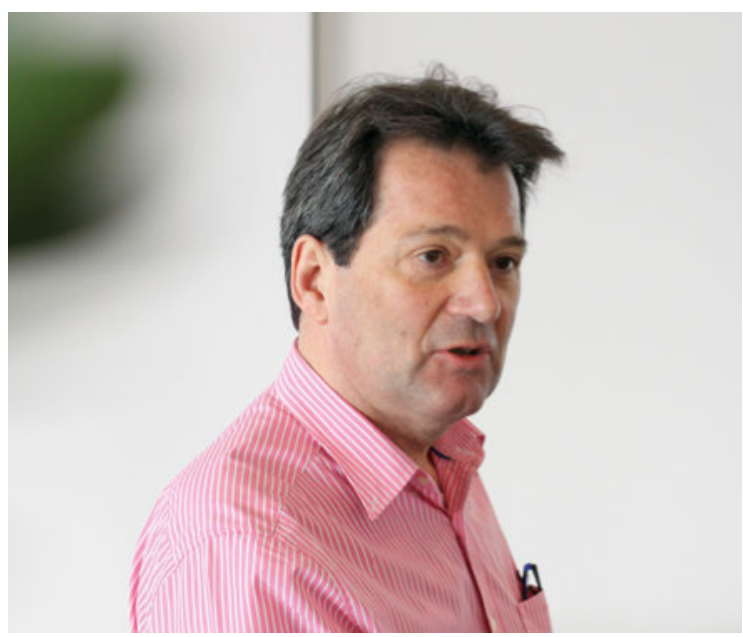

Setzte sich mit dem Ärztemangel auseinander: Laurent Bernheim, Vize-Dekan für Lehre an der Medizinischen Fakultät der Universität Genf.
Entsprechend lancierte die Gesundheitsdirektorenkonferenz die Idee einer interkantonalen Vereinbarung. Als sich abzeichnete, dass nicht alle Kantone daran teilnehmen, stiegen immer mehr Kantone aus der bestehenden Ostschweizer Vereinbarung aus. Die Solidarität kam an ihre Grenzen.

Derzeit ist offen, ob die interkantonale Vereinbarung zustande kommt, denn gemäss Bundesrecht müssen sich mindestens 18 Kantone daran beteiligen. Bis heute haben erst 13 Kantone die Vereinbarung ratifiziert. "Wir führen intensive Gespräche und hoffen immer noch, dass die Vereinbarung Anfang 2019 in Kraft treten kann", berichtete Hanselmann.

Unabhängig davon sei der Kanton St. Gallen auch auf anderen Ebenen aktiv geworden, um die medizinische Bildung zu stärken. Ab 2020 bieten die Universität St.Gallen und das Kantonsspital St.Gallen gemeinsam mit der Universität Zürich einen «Joint Medical Master» mit 40 Studienplätzen an. Finanziert wird das Angebot aus dem Sonderprogramm des Bundesrates im Rahmen seiner Strategie gegen den Ärztemangel.

\section{Medizinischer Röstigraben}

Mit dem Ärztemangel hat sich auch Laurent Bernheim, Vizedekan für Lehre an der Medizinischen Fakultät der Universität Genf, vertieft auseinandergesetzt. In seinem Referat kam er zu überraschenden Schlussfolgerungen. Auf der Grundlage des Berichts des Bundesrates von 2011 (Strategie gegen Ärztemangel und zur Förderung der Hausarztmedizin) berechnete er, dass in der Schweiz jährlich rund 1300 neue Ärztinnen und Ärzte ausgebildet werden sollten. Bernheim zeigte auf, dass in der Schweiz diesbezüglich ein Röstigraben besteht: In der Romandie werden genügend neue Ärztinnen und Ärzte ausgebildet 325 wären nötig, 400 werden ausgebildet. Anders in der Deutschschweiz und im Tessin - dort liegt der Bedarf bei 975 Personen, ausgebildet werden aber nur 720. Diesen Unterschied zwischen den Sprachregionen zu beachten sei wichtig, weil Ärztinnen und Ärzte den Röstigraben für eine berufliche Tätigkeit nur sehr selten überqueren.

Besteht in der Romandie somit kein Ärztemangel? Weit gefehlt, wie Laurent Bernheim weiter aufzeigte. Die Verteilung der Ärztinnen und Ärzte auf die einzelnen Fachgebiete stimme auch in der französischsprachigen Schweiz nicht mit dem Bedarf überein. Es mangle im Kanton Genf an Allgemeinmedizinern und gebe zu viele Fachärzte beispielsweise der Psychiatrie, Radiologie, orthopädischen Chirurgie und Kardiologie. Was tun? Die Aufwertung der Hausarztmedizin an den 


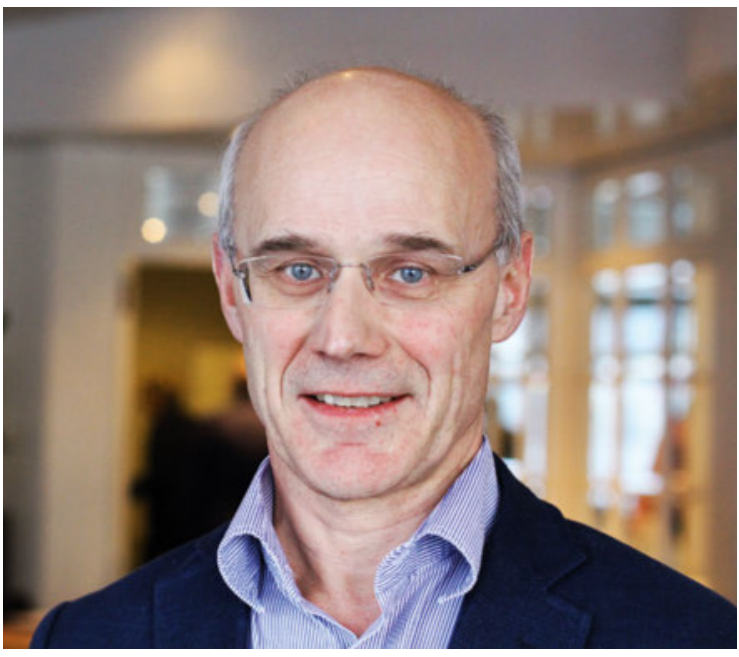

«E-Health wird nicht weniger, sondern mehr administrativen Aufwand mit sich bringen »: Jürg Lareida, Präsident des Aargauischen Ärzteverbandes.

Universitäten, finanzielle Anreize über den TARMED und Änderungen bei der Fortbildung werden nicht reichen, ist Bernheim überzeugt. Es werde auch einen gewissen Zwang brauchen, die jungen Ärzte in eine bestimmte Richtung zu lenken: «Ich bin nicht begeistert davon, aber wir sollten darüber nachdenken, bevor Politik und Krankenkassen uns dies aufzwingen.» Interessant in diesem Zusammenhang ist die Befragung der Ärztinnen und Ärzte in Weiterbildung, welche die ETH Zürich für das SIWF jeweils durchführt. In der Umfrage 2017 wurde unter anderem gefragt, ob

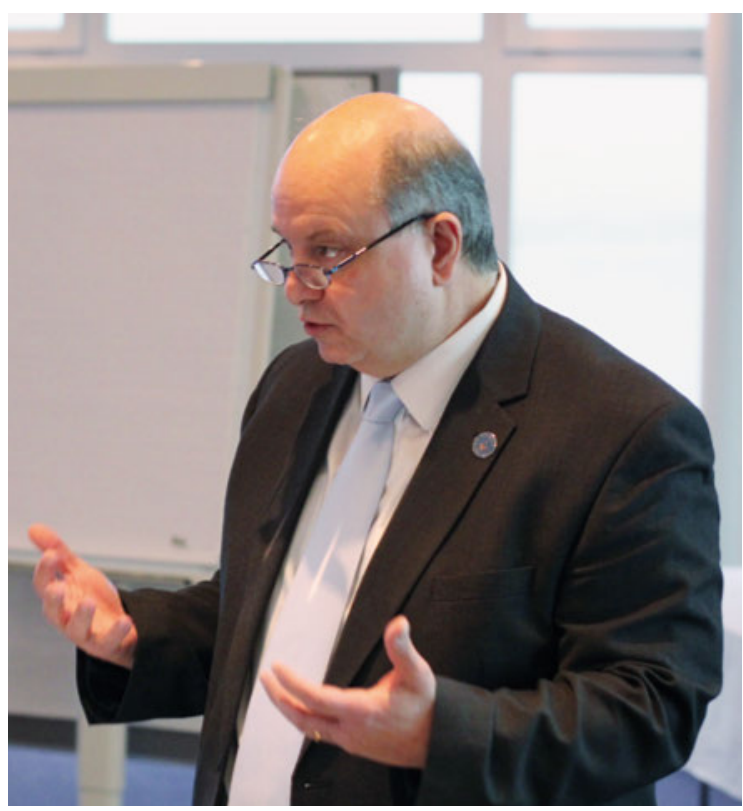

Plädierte für europaweite Qualitätsstandards: Vassilios Papalois, Generalsekretär der Union Européenne des Médecins Spécialistes (UEMS) man eine Steuerung der Anzahl Ärzte, die pro Fachrichtung weitergebildet werden, befürwortet. Die Leiterinnen und Leiter der Weiterbildungsstätten sprachen sich mehrheitlich dagegen aus (52 Prozent Nein, 40 Prozent Ja, 8 Prozent «Weiss nicht»). Anders sah es bei den Assistenzärztinnen und -ärzten aus: 38 Prozent befürworteten eine gezielte Steuerung, 32 Prozent waren dagegen und 30 Prozent hatten keine Meinung zu dieser Frage.

\section{Praktiker einbeziehen}

«Der Bedarf an Ärzten in der Praxis wird weiter zunehmen», zeigte sich Jürg Lareida, Präsident des Aargauischen Ärzteverbandes, in seinem Referat überzeugt. Der Aargauische Ärzteverband beispielsweise habe zwar seit 1994 seine Mitgliederzahl verdoppeln können. Viele Neueintretende - zumeist aus dem Ausland - seien allerdings bereits dem Pensionsalter nah. Wenn es in Zukunft immer häufiger an Arztpraxen mangle, bestehe die Gefahr, dass die Spitäler von Patienten überhäuft werden, sagte Lareida. Die demographische Entwicklung verlange nach mehr Ärzten, in der Realität aber geschehe das Gegenteil: Zunehmende administrative Arbeiten und häufigere Teilzeitpensen insbesondere von Ärztinnen verringerten die verfügbare Zeit für die Patienten. "Dass die administrative Belastung abnehmen wird, ist Wunschdenken", so Lareida. Schon jetzt zeige sich, dass etwa E-Health nicht weniger, sondern noch mehr administrativen Aufwand mit sich bringe.

Einen Lösungsansatz sieht Lareida darin, die Ausbildung flexibler zu gestalten - mit einem modularen Aufbau. So könnten die angehenden Ärzte selber bestimmen, wie schnell sie die einzelnen Lernziele erreichen wollen. Wichtig wäre zudem, die niedergelassenen Ärztinnen und Ärzte mehr einzubeziehen, etwa durch Weiterbildung in den Arztpraxen.

\section{Europäische Standards schaffen}

Was in der Schweiz ein Thema ist, beschäftigt auch Europa, wie Vassilios Papalois in seinem Referat aufzeigte. Der Professor für Transplantationschirurgie ist auch Generalsekretär der Union Européenne des Médecins Spécialistes (UEMS), des Dachverbandes der nationalen medizinischen Gesellschaften. Bis 2020 werden gemäss Europäischer Kommission eine Million Fachkräfte im Gesundheitswesen fehlen, sagte Papalois. Im Gegensatz zum innerschweizerischen Röstigraben sind die Ärztinnen und Ärzte sowie die Patienten innerhalb Europas sehr mobil, zeigte Papalois auf. Gemeinsame Qualitätsstandards in der medizinischen 


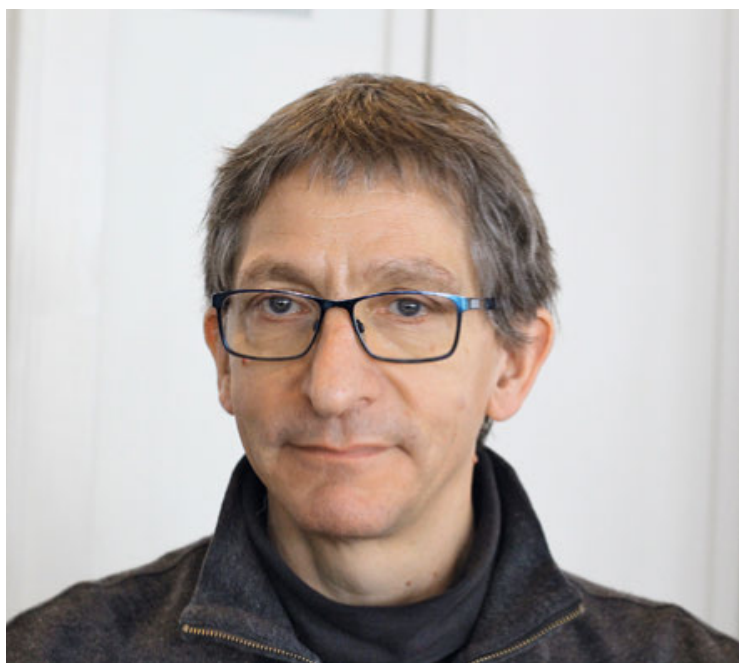

Die Digitalisierung nutzen lernen: Christian Lovis, Professor für Medizinische Informatik an der Universität Genf.

Bildung seien deshalb sehr wichtig. Bis heute existieren in vielen europäischen Ländern noch keine oder nur rudimentäre Assessments für die medizinische Aus- und Weiterbildung.

\section{Vorteile der Digitalisierung nutzen}

Zwei Workshops an der Journée de réflexion widmeten sich der Kontinuität der ärztlichen Bildung und der "Medizin 4.0». Geleitet wurde der letztere Workshop von Christian Lovis, Professor für Medizinische Informatik an der Universität Genf und Leiter der Division of Medical Information Sciences an den Universitätsspitälern Genf. Er erläuterte, dass die Medizin seit jeher ein hochgradig regulierter Bereich sei - von der Ausbildung bis zu den Medikamenten. Mit der Digitalisierung sei ein zusätzlicher, unregulierter Markt entstanden. So könnten Patienten heutzutage beispielsweise Bilder ihrer Krankheitssymptome online hochladen und erhalten eine Diagnose, die mittels Algorithmen zur Bildauswertung erstellt wurde. Das Ganze für manchmal nur einen Dollar!

Christian Lovis plädierte für einen pragmatischen, realistischen Ansatz. Noch sei schwierig abzuschätzen, inwiefern die Digitalisierung die Medizin verbessern helfe - sie werde Vor- und Nachteile mit sich bringen. Nötig sei aber, dass Mediziner die neuen Technologien in der Aus- und Weiterbildung kennen, aus einem wissenschaftlichen Blickwinkel beurteilen und anwenden lernen. Um die Vorteile der digitalen Medizin nutzen zu können, gelte es gerade in der Schweiz noch Hindernisse zu beseitigen. Die oft fehlende Kompatibilität der Daten sei weniger ein technisches als ein semantisches Problem. Der Austausch von Daten sei nur möglich, wenn alle dieselben präzisen Begriffe verwenden. Entsprechende Instrumente der Interoperabilität wie die Referenzdatenbank SNOMED würden in der Schweiz im Vergleich zum Ausland noch zu wenig genutzt.

\section{Gemeinsame Module}

Im zweiten Workshop ging es um die Frage, wie die Kontinuität zwischen der Aus- und der Weiterbildung verbessert werden kann. Dabei wurde unter anderem die Idee von Modulen diskutiert, die sowohl für Studierende wie Personen in der Weiterbildung wählbar wären. Insbesondere die «Entrustable Professional Activities» (EPAs) hätten das Potential, die Kontinuität zwischen Aus- und Weiterbildung zu verbessern, so die Rückmeldung aus dem Workshop ins Plenum.

Zum Abschluss einer Journée de réflexion wird jeweils eine To-do-Liste bis zum nächstjährigen Event erstellt. Einige Themen vom vergangenen Jahr haben weiteren Diskussions- und Handlungsbedarf und bleiben auf der Liste. Hier eine Auswahl: Die Lernziele überprüfen, die Anzahl der Weiterbildungsstellen und die Fachärzte-Verteilung diskutieren, die Finanzierung der Weiterbildung sicherstellen, die Weiterbildung vermehrt in Netzwerken organisieren sowie die Frage der Übernahme ärztlicher Tätigkeiten durch andere Fachpersonen. In der Schlussdiskussion wurde beschlossen, bis zur nächsten Journée de réflexion den Schwerpunkt auf folgende Frage zu setzen: Wie kann die zu grosse Trennung zwischen Ausbildung und Weiterbildung überwunden werden? Das Collège des doyens und das Institut für ärztliche Weiter- und Fortbildung (SIWF) wollen dazu in den kommenden Monaten auch das Gespräch mit den Fachgesellschaften und den medizinischen Fakultäten suchen. 